\title{
Request-based Dynamic Bandwidth Allocation of Gigabit Passive Optical Network
}

\author{
Chih-Ta Chiu, Yung-Chung Wang \\ Department of Electrical Engineering, National Taipei University of Technology, Taipei, Chinese Taipei \\ Email: ycwang@ee.ntut.edu.tw
}

Received 2013

\begin{abstract}
We propose request-based dynamic bandwidth allocation (DBA) of gigabit passive optical network (GPON). The optical line terminal (OLT) in GPON grants bandwidth to optical network units (ONUs). ONUs report request bandwidth which depends on queue lengths of traffic containers (TCONTs) to the OLT. In the OLT, DBA of GPON supports a request-based polling order to allocate bandwidth. Our request-based dynamic bandwidth allocation focuses on weight assignments in the request-based polling order. Weight assignments allocate bandwidth in proportion to guaranteed and request bandwidth. We use the $\mathrm{C}$ program to simulate results. Simulated results indicate improved performance in queueing delay when total offered loads are or are not shared uniformly to TCONTs.
\end{abstract}

Keywords: GPON; DBA; Weight; Polling

\section{Introduction}

GPON [1], a network of data transmission between the optical line terminal (OLT) and optical network units (ONUs), provides point to multipoint (P2MP) broadband access networks. To achieve P2MP, tree topology [2] gives a high distance extension solution because low lengths of fiber links make a low cost. In the upstream direction, time-division multiplexing access (TDMA) [3] is used. ONUs send packets in their own time slots to the OLT. In the downstream direction, the OLT broadcasts packets in the downstream direction to ONUs. Headers of packets not only make the OLT assign time slots to ONUs but also make ONUs report their queue lengths to the OLT. By reporting queue lengths, time slots are assigned dynamically.

DBA [4], based on the TDMA protocol, improves performance compared with performance of the static bandwidth allocation. DBA is achieved by header fields of packets [5]. Header fields in the downstream direction includes allocation identifiers (AllocIDs) to identify TCONTs, start time pointers to denote the start time of time slots, and end time pointers to denote the end time of time slots. Those fields specify that only one of TCONTs is served at any time. Header fields in the upstream direction include AllocIDs to identify TCONTs and dynamic bandwidth reports (DBRs) to report queue lengths in TCONTs. DBRs notifies the OLT to allocate bandwidth dynamically due to queue lengths of ONUs are different. Bandwidth is allocated dynamically to sat- isfy queue lengths as soon as possible so that performance is improved.

The literature of DBA is rich. Instead of constant time slots, [6] achieves dynamic time slots. Headers in the upstream and downstream direction are used to grant bandwidth and report queue lengths, respectively. The time interval within a time slot only depends on reported queue lengths. Based on service level agreement (SLA), [7] reserves a guaranteed and non-guaranteed bandwidth allocation policy. Bandwidth is cut into multiple grids which includes guaranteed or non-guaranteed bandwidth. Then, grids are polled for ONUs so that the policy is achieved. [8] determines which ONU is allocated guaranteed or non-guaranteed bandwidth. A call admission control (CAC) generates characters of ONUs as non-guaranteed, guaranteed, or delay guaranteed bandwidth. Then, by evenly delay algorithm, ONUs are put in grids. Then, by BGP, bandwidth is polled from the first to the last grid for ONUs. [9] provides a multimedia service by allocating high to low priority bandwidth called fixed, assured, and best effort bandwidth. In [10] and [11], a class-based bandwidth scheme provides the differentiated service because minimum function in the OLT compares critical values with request bandwidth to choose a smaller one. The OLT allocates bandwidth to queues of different traffic classes. Then, ONUs report queue lengths according to traffic classes. [12] sets inter-ONU and intra-ONU weights so that bandwidth can be allocated in proportion to weights. Types of the scheduling can be weighted or hierarchical round robin. 
In the DBA called prioritized weighted round robin (PWRR) [5] [13], four kinds of bandwidth from high to low priority bandwidth are fixed, assured, non-assured, and best-effort bandwidth. Fixed and assured bandwidth is guaranteed bandwidth while non-assured bandwidth and best-effort bandwidth is surplus bandwidth. Critical values include maximum and minimum bandwidth. Critical values of non-assured bandwidth is in proportion to critical values of assured bandwidth. The minimum function compares request bandwidth with critical values to choose a smaller one. Polling types are weighted round robin (WRR). Time slots for bandwidth of four priorities bandwidth are allocated every 125 us. [14] gives different offered loads in its simulation to evaluate performance of DBA.

We propose the request-based DBA called prioritized adaptive request-based polling (PARP) in GPON. PARP includes weight assignments and request polling. In weight assignments, non-assured bandwidth is allocated in proportion to weights. Two parameters are adaptable for weights which are in proportion to assured and request bandwidth. Mention to the request-based polling, instead of the fixed polling order of WRR, polling order in PARP is based on the highest request bandwidth among all request bandwidth within the same type of TCONTs. Therefore, each type of TCONTs has its own polling order. PARP is based on request bandwidth to achieve DBA and polling. We use a $\mathrm{C}$ program to simulate results. Simulate results improve performance in queueing delay when total offered loads are or are not shared uniformly to TCONTs.

\section{Proposed Method}

\subsection{Operations}

GPON shown in Figure 1 includes OLT, ONUs and the optical distribution network (ODN). OLT is responsible for allocating bandwidth while ONUs are responsible for reporting their queue lengths. ODN connects between the OLT and ONUs to achieve broadcast in the downstream direction and TDM in the upstream direction. Queues in ONUs are TCONTs with queue lengths. Each TCONT is identified by an AllocID so that the OLT can use AllocIDs to identify TCONTs.

Relationships between TCONTs and bandwidth are shown in Figure 2. TCONT1 is allocated fixed bandwidth which does not consider request bandwidth. TCONT2 is allocated assured bandwidth. TCONT3 is allocated assured and non-assured bandwidth. TCONT4 is allocated best-effort bandwidth. TCONT5 is the type of test queues so that it is allocated fixed, assured, nonassured, best-effort, and combined bandwidth. Priorities to allocate bandwidth are shown in Figure 3. Fixed and assured bandwidth is guaranteed bandwidth while non- assured bandwidth and best-effort bandwidth is surplus bandwidth. Order from high to low priority is fixed, assured, non-assured, and best-effort bandwidth.

\subsection{Assumptions}

In [13], DBA for TCONT 3 is specified:

- For the surplus bandwidth allocation to TCONT 3, setting all SImin parameters equal and varying the ABsur parameters, a weighted round robin service is enforced. If all the ABsur parameters are also set equal, the service becomes equivalent to a simple round robin.

- The guaranteed service rate is expressed as: $A B$ $\min / S I m a x$, while the surplus (non-guaranteed) service rate is expressed as: ABsur/SImin. The guaranteed rate and the surplus rate sum up to the allowed peak rate for TCONT 3 .

- $\quad$ Regarding TCONT 3 AllocIDs, a GIR $=1 / 3 P I R$ was used.

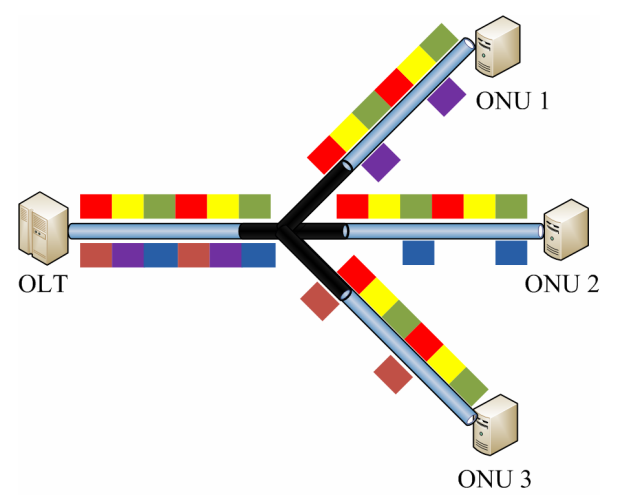

Figure 1. GPON.

\begin{tabular}{|c|c|c|c|c|c|}
\cline { 2 - 6 } \multicolumn{1}{c|}{} & TCONT1 & TCONT2 & TCONT3 & TCONT4 & TCONT5 \\
\hline $\begin{array}{c}\text { Fixed } \\
\text { bandwidth }\end{array}$ & $\mathrm{Y}$ & $\mathrm{N}$ & $\mathrm{N}$ & $\mathrm{N}$ & $\mathrm{Y}$ \\
\hline $\begin{array}{c}\text { Assured } \\
\text { bandwidth }\end{array}$ & $\mathrm{N}$ & $\mathrm{Y}$ & $\mathrm{Y}$ & $\mathrm{N}$ & $\mathrm{Y}$ \\
\hline $\begin{array}{c}\text { Non assured } \\
\text { bandwidth }\end{array}$ & $\mathrm{N}$ & $\mathrm{N}$ & $\mathrm{Y}$ & $\mathrm{N}$ & $\mathrm{Y}$ \\
\hline $\begin{array}{c}\text { Best effort } \\
\text { bandwidth }\end{array}$ & $\mathrm{N}$ & $\mathrm{N}$ & $\mathrm{N}$ & $\mathrm{Y}$ & $\mathrm{Y}$ \\
\hline $\begin{array}{c}\text { Combined } \\
\text { bandwidth }\end{array}$ & $\mathrm{N}$ & $\mathrm{N}$ & $\mathrm{N}$ & $\mathrm{N}$ & $\mathrm{Y}$ \\
\hline
\end{tabular}

Figure 2. Relationships between bandwidth and TCONTs.

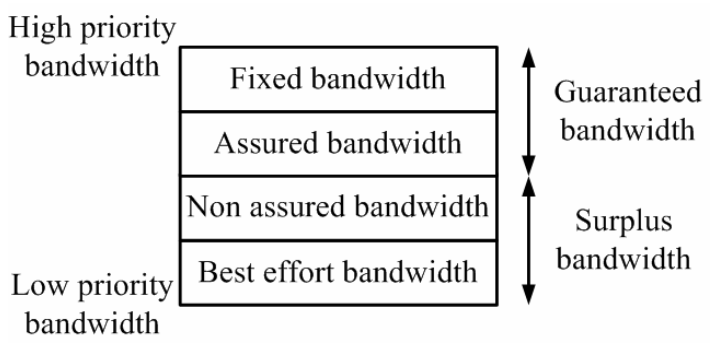

Figure 3. Priorities of the bandwidth allocation. 
To compare our proposed method with PWRR, we assume that PWRR in this paper sets all SImax and SImin parameters equal and varies ABsur parameters so that a WRR service is enforced. We follows the GIR $=1 / 3 P I R$. Because the peak information rate $(P I R)$ is three times of guaranteed information rate (GIR), with setting SImax and SImin equal, $A B \min =1 / 2 A B s u r$ is used for TCONT3. In this paper, $A B m i n$ and $A B s u r$ for TCONT3 is $B_{\max }^{T C O N T 3}$ and $B_{\min }^{T C O N T 3}$, respectively.

\subsection{Prioritized Adaptive Weighted Round Robin}

We propose prioritized adaptive weighted round robin (PAWRR) with weight assignments and without requestbased polling. Equation (1) and (2) are maximum bandwidth. Maximum bandwidth is critical values. Maximum bandwidth of TCONT2 is determined by OfferedLoad $^{\text {TCONT2 }}$ while maximum bandwidth of TCONT3 is determined by OfferedLoad ${ }^{\text {TCONT3 }}$. Equation (3) and (4) are assured bandwidth. Assured bandwidth of TCONT2 and TCONT3 is allocated by comparing request bandwidth with maximum bandwidth to choose a smaller one.

$$
\begin{aligned}
& B_{\max }^{\text {TCONT2 }}=\text { OfferedLoad }^{\text {TCONT2 }} . \\
& B_{\max }^{\text {TCONT3 }}=\text { OfferedLoad }^{\text {TCONT3 }} . \\
& B_{\text {assured }}^{\text {TCONT2 }}=\min \left(B_{\text {request }}^{\text {TCONT2 }}, B_{\max }^{\text {TCONT2 }}\right) . \\
& B_{\text {assured }}^{\text {TCONT3 }}=\min \left(B_{\text {request }}^{\text {TCONT3 }}, B_{\max }^{\text {TCONT3 }}\right) .
\end{aligned}
$$

Equation (5), (6), and (7) are surplus, minimum bandwidth, and weight assignments, respectively. Surplus bandwidth is allocated for non-assured bandwidth by weights of TCONT3. Weights of TCONT3 are assigned in proportion to assured bandwidth and request bandwidth. Parameters of $\alpha$ and $\beta$ are the influence of assured and request bandwidth, respectively. The sum of $\alpha$ and $\beta$ is 1.0. Equation (8) is non-assured bandwidth. Non-assured bandwidth is allocated by choosing the smallest one among request bandwidth, minimum bandwidth, and frame bytes bandwidth. Frame bytes bandwidth is unused bandwidth after allocating bandwidth.

$$
\begin{aligned}
& B_{\text {surplus }}=B_{\text {total }}-\left(\sum_{i=0}^{N^{T C O N T 2}} B_{i, \text { assured }}^{\text {TCONT2 }}+\sum_{i=0}^{N^{T C O N T 3}} B_{i, \text { assured }}^{T C O N T 3}\right) \text {. } \\
& B_{\min }^{\text {TCONT3 }}=B_{\text {surplus }} \times \frac{W^{\text {TCONT3 }}}{\sum_{i=0}^{N_{\text {TCONT3 }}} W_{i}^{\text {TCONT3 }}} . \\
& W^{\text {TCONT3 }}=\frac{\alpha \times B_{\text {assured }}^{\text {TCONT3 }}+\beta \times B_{\text {request }}^{\text {TCONT3 }}}{\sum_{i=0}^{N^{T C O N T 3}}\left(\alpha \times B_{i, \text { assured }}^{T C O N T 3}+\beta \times B_{i, \text { request }}^{T C O N T 3}\right)} . \\
& B_{\text {non-assured }}^{T C O N T 3}=\min \left(B_{\text {request }}^{T C O N T 3}, B_{\min }^{T C O N T 3}, B_{\text {frame-bytes }}\right) \text {. }
\end{aligned}
$$

Equation (9) and (10) are minimum and best-effort bandwidth, respectively. Best-effort bandwidth is allocated by comparing request bandwidth with frame bytes bandwidth to choose a smaller one.

$$
\begin{gathered}
B_{\min }^{\text {TCONT4 }}=B_{\text {frame-bytes }} \cdot \\
B_{\text {best-effort }}^{\text {TCONT4 }}=\min \left(B_{\text {request }}^{\text {TCONT }}, B_{\min }^{\text {TCONT4 }}\right) .
\end{gathered}
$$

\subsection{Prioritized Adaptive Request-based Polling}

We proposed prioritized adaptive request-based polling (PARP) with weight assignments and request-based polling. Figure 4 is the scheme of request-based polling. Instead of a count-down timer, we use the 0-1 trigger called SImax or SImin to determine which TCONT can be allocated bandwidth. When a TCONT can and can not allocate bandwidth, trigger value is 1 and 0 , respectively.

In the beginning, we copy requests of $N$ TCONT2, 3, and 4 as TempTCONT2, TempTCONT3, and TempTCONT4 in number $u$ time slot, respectively. Then, we use MatchTCONT2, MatchTCONT3, and MatchTCONT4 to choose the highest request bandwidth from TempTCONT2, TempTCONT3, and TempTCONT4, respectively. If MatchTCONT2 is 0 , the polling order follows the order of weighted round robin due to all request bandwidth of TCONT2 is zero for no comparison. If MatchTCONT2 is not 0 , the polling order is the highest request bandwidth TCONT2. After MatchTCONT2, it turns to MatchTCONT3. If MatchTCONT3 is 0, the polling order follows the order of weighted round robin due to all request bandwidth of TCONT3 is zero for no comparison. If MatchTCONT3 is not 0 , the polling order is the highest

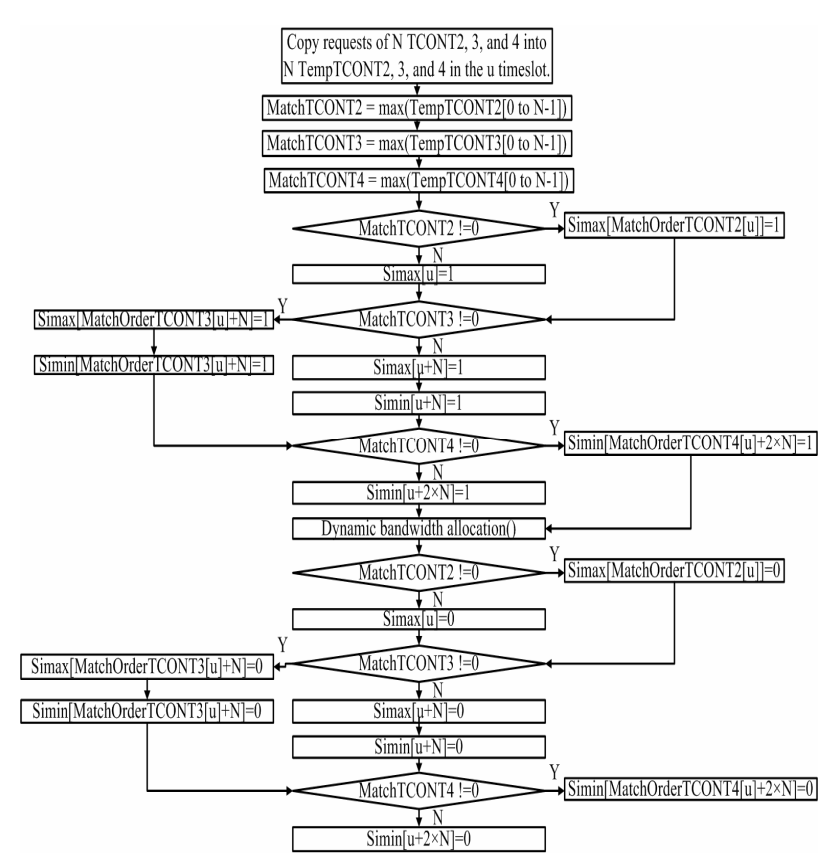

Figure 4. Request-based polling. 
request bandwidth TCONT3. After MatchTCONT3, it turns to MatchTCONT4. If MatchTCONT4 is 0, the polling order follows the order of weighted round robin due to all request bandwidth of TCONT4 is zero for no comparison. If MatchTCONT4 is not 0 , the polling order is the highest request bandwidth TCONT4. Simax and Simin follow the position of MatchTCONT2, MatchTCONT3, and MatchTCONT4 to set its value as 1 for the bandwidth allocation. Then, DBA works for the bandwidth allocations of TCONTs whose SImax or SImin is 1 . After DBA, its value is set 0 .

\subsection{Examples}

The example includes one ODN between three ONUs and one OLT. Each ONU includes three TCONTs which are one TCONT2, one TCONT3, and one TCONT4. The OLT includes a table about request bandwidth shown in Figure 5. In this example, we use parameters in unit of bytes. $B_{\text {frame-bytes }}$ comes from $125 \times 10^{3} / 8 / 1=15625$ bytes. OfferedLoad ${ }^{\mathrm{TCONT} 2}$ is 6000 bytes and OfferedLoad $^{\text {TCONT3 }}$ is 3000 bytes so that $B_{\max }^{\text {TCONT2 }}$ is 6000 bytes and $B_{\max }^{\text {TCONT3 }}$ is 3000 bytes. Request bytes of three TCONTs of ONU1, ONU2, and ONU3 are 9000, 6000, and 3000 bytes, respectively. The PARP table is observed within three frame bytes. In this example, guard bytes are ignored, pre-assured bandwidth of TCONT2 and 3 is 3000 bytes, and $\beta$ is 1.0 .

In the first frame bytes, firstly, $\max (9000,6000,3000)$ $=9000$ bytes so that TCONT 2 of ONU1 is chosen. In the first frame bytes, request bytes of TCONT2 of ONU1 decrease from 9000 bytes to 3000 bytes due to $\min (9000$, $6000)=6000$ bytes for the assured bandwidth allocation. Remainders of frame bytes decrease from 15625 bytes to 9625 bytes.

\begin{tabular}{|c|c|c|c|c|}
\hline Table & ONU 1 & ONU 2 & ONU 3 & $\begin{array}{c}\text { Remainders of } \\
\text { frame bytes }\end{array}$ \\
\hline TCONT 2 & 9000 & 6000 & 3000 & 15625 \\
\hline TCONT 3 & 9000 & 6000 & 3000 & 15625 \\
\hline TCONT 4 & 9000 & 6000 & 3000 & 15625 \\
\hline \hline $\begin{array}{c}\text { After 1 } \\
\text { bytes of PARP }\end{array}$ & ONU 1 & ONU 2 & ONU 3 & $\begin{array}{c}\text { Remainders of } \\
\text { frame bytes }\end{array}$ \\
\hline TCONT 2 & 3000 & 6000 & 3000 & 9625 \\
\hline TCONT 3 & 0 & 6000 & 3000 & 625 \\
\hline TCONT 4 & 8375 & 6000 & 3000 & 0 \\
\hline $\begin{array}{c}\text { After } 2^{\text {nd }} \text { frame } \\
\text { bytes of PARP }\end{array}$ & ONU 1 & ONU 2 & ONU 3 & $\begin{array}{c}\text { Remainders of } \\
\text { frame bytes }\end{array}$ \\
\hline TCONT 2 & 3000 & 0 & 3000 & 9625 \\
\hline TCONT 3 & 0 & 0 & 3000 & 3625 \\
\hline TCONT 4 & 4750 & 6000 & 3000 & 0 \\
\hline \hline $\begin{array}{c}\text { After } 3^{\text {rd }} \text { frame } \\
\text { bytes of PARP }\end{array}$ & ONU 1 & ONU 2 & ONU 3 & $\begin{array}{c}\text { Remainders } \\
\text { of frame bytes }\end{array}$ \\
\hline TCONT 2 & 0 & 0 & 3000 & 12625 \\
\hline \begin{tabular}{c} 
TCONT 3 \\
\hline TCONT 4
\end{tabular} & 0 & 0 & 0 & 9625 \\
\hline
\end{tabular}

Figure 5. DBA with PARP.
Secondly, $\max (9000,6000,3000)=9000$ bytes so that TCONT3 of ONU1 is chosen. Request bytes of TCONT3 of ONU1 decrease from 9000 bytes to 6000 bytes due to $\min (9000,3000)=3000$ bytes for the assured bandwidth allocation. Remainders of frame bytes decrease from 9625 bytes to 6625 bytes. Request bytes of TCONT3 of ONU1 decrease from 6000 bytes to 0 byte from (15625 $\times 3-6000-3000 \times 5) \times 6000 /(6000+6000+3000)=$ 10350 bytes and $\min (6000,10350,6625)=6000$ bytes for the non-assured bandwidth allocation. Remainders of frame bytes decrease from 6625 bytes to 625 bytes.

Thirdly, $\max (9000,6000,3000)=9000$ bytes so that TCONT4 of ONU1 is chosen. Request bytes of TCONT4 of ONU1 decrease from 9000 bytes to 8375 bytes due to $\min (9000,625)=625$ bytes for the best-effort bandwidth allocation.

In the second frame bytes, firstly, TCONT2 of ONU2 is chosen due to $\max (3000,6000,3000)=6000$ bytes which turns to TCONT2 of ONU2. Firstly, in PARP table of the second frame bytes, request bytes of TCONT2 of ONU2 decrease to 0 bytes for the assured bandwidth allocation due to $\min (6000,6000)=6000$ bytes. Remainders of frame bytes decrease from 15625 bytes to 9625 bytes.

Secondly, $\max (0,6000,3000)=6000$ bytes so that TCONT3 of ONU2 is chosen. Request bytes of TCONT3 of ONU2 decrease from 6000 bytes to 3000 bytes due to $\min (6000,3000)=3000$ bytes for the assured bandwidth allocation. Then, request bytes of TCONT3 of ONU1 decrease from 3000 bytes to 0 bytes from $(15625 \times 3$ $6000-3000 \times 5) \times 6000 /(0+6000+3000)=17250$ bytes and $\min (3000,17250,6625)=3000$ bytes for the non-assured bandwidth allocation. Remainders of frame bytes decrease from 6625 bytes to 3625 bytes.

Thirdly, TCONT4 of ONU1 is chosen due to $\max (8375,6000,3000)=8375$ bytes. Request bytes of TCONT4 of ONU1 decreases from 8375 bytes to 4750 bytes due to $\min (8375,3625)=3625$ bytes for the best effort bandwidth allocation. Remainders of frame bytes decrease from 3625 bytes to 0 byte.

In the third frame bytes, firstly, due to $\max (3000,0$, $3000)=3000$ bytes, it turns to TCONT2 of ONU1. In PARP table of the third frame bytes, request bytes of TCONT2 of ONU1 decrease from 3000 bytes to 0 byte due to $\min (3000,6000)=3000$ bytes for assured bandwidth allocation. Remainders of frame bytes decrease from 15625 bytes to 12625 bytes.

Secondly, due to $\max (0,0,3000)=3000$ bytes, TCONT3 of ONU3 is chosen. Request bytes of TCONT3 of ONU3 decrease from 3000 bytes to 0 byte. Remainders of frame bytes decrease from 12625 bytes to 9625 bytes due to $\min (3000,3000)=3000$ bytes for the assured bandwidth allocation.

Thirdly, due to $\max (4750,6000,3000)=6000$ bytes, 
TCONT4 of ONU2 is allocated best-effort bandwidth. Request bytes of TCONT4 of ONU2 decrease from 6000 bytes to 0 byte due to $\min (6000,9625)=6000$ bytes . Remainders of frame bytes decrease from 9625 bytes to 3625 bytes. PARP allocates high request bytes.

\section{Performance Evaluation}

\subsection{Parameters}

GPON is simulated in a $\mathrm{C}$ program to evaluate performance of PARP with 1 OLT and 8 ONUs. Mention to simulated parameters, bandwidth of GPON is set as 1.24416 Gbps and network capacity is set as $1 \mathrm{G}$. The frame duration is set as 125 us. Propagation delay between OLT and ONUs is set as 200 us. Guard bytes are set as 20 bytes. The size of TCONTs is set as 10 Mbytes. TCONT types are one TCONT2, one TCONT3, and one TCONT4 in each ONU. The network traffic is generated by exponential inter-arrival time and packet sizes are 64 bytes, 500 bytes, and 1500 bytes with the probability 0.6 , 0.2 , and 0.2 , respectively. Parameters of OfferedLoad ${ }^{\text {TCONT2 }}$ and OfferedLoad ${ }^{\text {TCONT3 }}$ are 9500 bytes and 3000 bytes, respectively. In the scenariol of queueing delay with sharing uniformly total offered loads, total offered loads are shared $1 / 24$ to each of 24 TCONTs in 8 ONUs. In the scenario 2 of queueing delay without sharing uniformly total offered loads, total offered loads are shared $1 / 16$ to each of 12 TCONTs in 4 ONUs and $1 / 48$ to each of 12 TCONTs in 4 ONUs.

\subsection{Scenario1}

Figure 6 is the comparison of queueing delay when total offered loads are shared uniformly to TCONTs. In the $1.7 \mathrm{Gbps}$ total offered load, four kinds of queueing delay of TCONT3 of PAWRR with setting $\alpha$ 1.0, PAWRR with setting $\beta$ 1.0, PARP, and PWRR are $3.97 \mathrm{~ms}$, $3.70 \mathrm{~ms}, 3.62 \mathrm{~ms}$, and $3.80 \mathrm{~ms}$, respectively.

Compared with $3.97 \mathrm{~ms}$ and $3.80 \mathrm{~ms}$ queueing delay, $B_{\min }^{\text {TCONT3 }}$ of TCONT3 of PAWRR with setting $\alpha 1.0$ is low since heavy loads make $B_{\text {surplus }}$ low and $B_{\text {assured }}^{\text {TCONT3 }}$ fixed. Fixed $B_{\text {assured }}^{\text {TCONT3 }}$ makes $W^{\text {sCONT3 }}$ fixed. $B_{\min }^{\text {TCOST3 }}$ of TCONT3 of PWRR is set only in proportion to $B_{\max }^{\text {TCONT3 }}$ which is a critical value of assured bandwidth. Therefore, $B_{\min }^{T C O N T 3}$ of TCONT3 of PAWRR is lower than $B_{\min }^{T C O N T 3}$ of TCONT3 of PWRR. $B_{\text {non-assured }}^{\text {TCONT3 }}$ is allocated more to TCONT3 of PWRR than TCONT3 of PAWRR setting $\alpha 1.0$.

Compared with $3.80 \mathrm{~ms}$ and $3.70 \mathrm{~ms}$ queueing delay, $B_{\min }^{\text {TCONT3 }}$ of TCONT3 of PAWRR with setting $\beta \quad 1.0$ is high to high request bandwidth TCONT3 and low to low request bandwidth TCONT3 since the weights are set in proportion to $B_{\text {request }}^{\text {TCONT3 }}$. Even $B_{\min }^{\text {TCONT3 }}$ of TCONT3 of PWRR is high, low $B_{\text {request }}^{\text {TCONT3 }}$ is chosen by the minimum function and high $B_{\text {request }}^{\text {TCONT3 }}$ needs more bandwidth than $B_{\min }^{T C O N T 3}$. Therefore, $B_{\text {non-assured }}^{T C O N 3}$ is allocated more to TCONT3 of PAWRR with setting $\beta \quad 1.0$ than TCONT3 of PWRR.

Compared with $3.70 \mathrm{~ms}$ and $3.62 \mathrm{~ms}$ queueing delay, WRR of PAWRR follows a fixed polling order. When request bandwidth is low, the request bandwidth is still polled. This results in low bandwidth allocation from a minimum function. PARP chooses highest request bandwidth to be polled. Therefore, more bandwidth is allocated due to request bandwidth is high.

\subsection{Scenario 2}

Figure 7 is the comparison of queueing delay when total offered loads are not shared uniformly to TCONTs. In the $1 \mathrm{Gbps}$ total offered load, four kinds of queueing delay of TCONT3 of PAWRR with setting $\alpha$ 1.0, PAWRR with setting $\beta$ 1.0, PARP, and PWRR are $5.18 \mathrm{~ms}, 5.12 \mathrm{~ms}, 1.82 \mathrm{~ms}$, and $8.17 \mathrm{~ms}$, respectively.

Compared with $5.18 \mathrm{~ms}$ and $8.17 \mathrm{~ms}$ queueing delay, $B_{\text {surplus }}$ is high since $1 \mathrm{Gbps}$ is light loads for TCONT3. Therefore, $B_{\min }^{\text {TCONT3 }}$ of TCONT3 of PAWRR is higher than $B_{\min }^{\text {TCONT3 }}$ of TCONT3 of PWRR. $B_{\text {non-assured }}^{\text {TCOT3 in }}$ allocated more to TCONT3 of PAWRR with setting $\alpha$ 1.0 than TCONT3 of PWRR. Compared with $5.18 \mathrm{~ms}$ and $5.12 \mathrm{~ms}$ queueing delay, $B_{\min }^{\text {TCONT3 }}$ of TCONT3 of PAWRR with setting $\beta 1.0$ is high to high request bandwidth TCONT3 and low to low request bandwidth TCONT3 since the weights are set in proportion to $B_{\text {surplus }}$. Therefore, $B_{\text {non-assured }}^{\text {TCONT }}$ is allocated more to TCONT3 of PAWRR with setting $\beta \quad 1.0$ than TCONT3 of PAWRR with setting $\alpha$ 1.0.

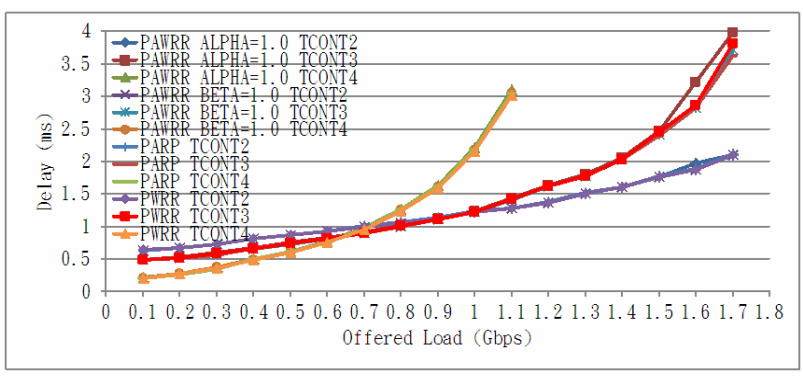

Figure 6. Performance of queueing delay in scenario1.

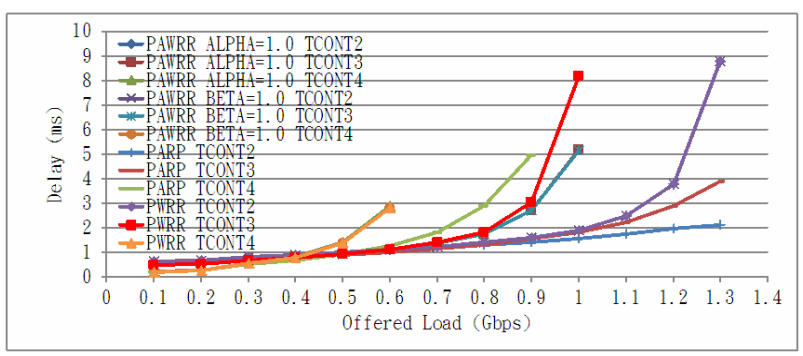

Figure 7. Performance of queueing delay in scenario2. 
Compared with $5.12 \mathrm{~ms}$ and $1.82 \mathrm{~ms}$ queueing delay, WRR in PAWRR follows a fixed polling order. Times to serve each TCONT is equal. However, TCONTs with high and less loads need more and less polling times, respectively. PARP gives more and less polling times to TCONTs with high and less loads, respectively. Therefore, queueing delay of PARP is lower than queueing delay of PAWRR with setting $\beta$ 1.0.

By different polling times, besides TCONT3, PARP improves queueing delay of TCONT2 and 4. In the 1.3 Gbps total offered load, four kinds of queueing delay of TCONT2 of PAWRR with setting $\alpha$ 1.0, PAWRR with setting $\beta$ 1.0, PARP, and PWRR are $8.76 \mathrm{~ms}$, $8.76 \mathrm{~ms}, 2.10 \mathrm{~ms}$, and $8.76 \mathrm{~ms}$, respectively. In the 0.6 Gbps total offered load, four kinds of queueing delay of TCONT4 of PAWRR with setting $\alpha 1.0$, PAWRR with setting $\beta$ 1.0, PARP, and PWRR are $2.87 \mathrm{~ms}$, $2.80 \mathrm{~ms}, 1.27 \mathrm{~ms}$, and $2.81 \mathrm{~ms}$, respectively.

\section{Conclusions}

We propose request-based DBA called PARP in GPON. PARP allocates min-max bandwidth with weights for critical values. When weights are assigned in proportion to request bandwidth, non-assured bandwidth is allocated more and less to high and less request bandwidth TCONT3, respectively. Non-assured bandwidth is adapted so that it adapts varying request bandwidth. Beside the bandwidth allocation, request-based polling is used to poll the highest request bandwidth TCONTs in the same type. PARP allocates more bandwidth to TCONTs of high request bandwidth. By a $\mathrm{C}$ program, simulations are evaluated when total offered loads are or are not uniformly shared to TCONTs. Simulative results indicate queueing delay in proportion to request bandwidth is better than one in proportion to guaranteed bandwidth and critical values. Simulative results also indicate queueing delay is improved when the polling order is chosen high request bandwidth TCONTs.

\section{REFERENCES}

[1] ITU Rec. G.984.1, "Gigabit-Capable Passive Optical Networks (G-PON): General Characteristics," Geneva, Switzerland, 2003.

[2] M. Mcgarry, M. Maier and M. Reisslein, "Ethernet Passive Optical Network (EPON): Building A Next-Generation Optical Access Network," IEEE Communications Magazine, Vol. 40, No. 2, 2002, pp. 66-73. doi:10.1109/35.983910

[3] D. Angelopoulos, S. Venieris and I. Stassinopoulos, "A TDMA Based Access Control Scheme for APON's," Journal of Lightwave Technology, Vol. 11, No. 5, 1993, pp. 1095-1103. doi:10.1109/50.233273

[4] ITU Rec. G.983.4, “A Broadband Optical Access System with Increased Service Capability Using Dynamic BandWidth Assignment," Geneva, Switzerland, 2001.

[5] J. Angelopoulos, H. Leligou, T. Argyriou and S. Zontos, "Efficient Transport of Packets with QoS in An FSAN-Aligned GPON," IEEE Communications Magazine, Vol. 42, No. 2, 2004, pp. 92-98. doi:10.1109/MCOM.2003.1267106

[6] G. Kramer, B. Mukherjee and G. Pesavento, "IPACT: A Dynamic Protocol for An Ethernet PON," IEEE Communications Magazine, Vol. 40, No. 2, 2002, pp. 74-80. doi:10.1109/35.983911

[7] M. Ma, Y. Zhu and T. Cheng, "A Bandwidth Guaranteed Polling MAC Protocol for Ethernet Passive Optical Networks," IEEE INFOCOM, Vol. 1, No. 30, 2003, pp. 22-31.

[8] Y. Zhu, M. Ma and T. Cheng, "A Novel Multiple Access Scheme for Ethernet Passive Optical Networks," IEEE GLOBECOM, Vol. 22, No. 1, 2003, pp. 2649-2653.

[9] S. Choi and J. Huh, "Dynamic Bandwidth Allocation Algorithm for Multimedia Services Over Ethernet PONs," IEEE INFOCOM, Vol. 1, No. 30, 2003, pp. 22-31.

[10] C. Assi, Y. Ye and S. Dixit, "Support of QoS in IP-based"

[11] Ethernet PON, IEEE GLOBECOM, Vol. 7, No. 1, 2003, pp. 3737-3741. doi:10.1080/028418501127346846

[12] J. Xie, S. Jiang and Y. Jiang, "A Dynamic Bandwidth Allocation Scheme for Differentiated Services in EPONs," IEEE Communications Magazine, Vol. 42, No. 8, 2004, pp. 32-39. doi:10.1109/MCOM.2004.1321385

[13] B. Chen, J. Chen and S. He, "Efficient and Fine Scheduling Algorithm for Bandwidth Allocation in Ethernet Passive Optical Networks," IEEE Journal of Selected Topics in Quanium Electronicsxxcta Radiologica, Vol. 12, No. 4, 2006, pp. 653-660. doi:10.1109/JSTQE.2006.876607

[14] H. Leligou, C. Linardakis, K. Kanonakis, J. Angelopoulos, and T. Orphanoudadkis, "Efficient Medium Arbitration of FSAN-Compliant GPONs," Journal of Communication Systems, Vol. 19, No. 5, 2006, pp. 603-607. doi:10.1002/dac.761

[15] C. Su-il and P. Jaehyung, "SLA-Aware Dynamic BandWidth Allocation for QoS in EPONs," IEEE/OSA Journal of Optical Communications and Networking, Vol. 2, No. 9, 2010, pp. 773-781.doi:10.1364/JOCN.2.00077 